\title{
Discrete Model of Electron
}

\author{
José Garrigues-Baixauli ${ }^{1}$ \\ ${ }^{1}$ Higher Technical School of Telecommunication Engineers, Spain. Retired professor from 2015. \\ Correspondence: José Garrigues-Baixauli, Universitat Politècnica de València, Camino de Vera s/n., 46022 \\ Valencia, Spain. E-mail: jgarrigu@eln.upv.es
}

Received: October 15, 2019

Accepted: November 20, 2019 Online Published: November 30, 2019

doi:10.5539/apr.v11n6p36

URL: http://dx.doi.org/10.5539/apr.v11n6p36

\begin{abstract}
An electron model is developed based on a 4D sphere with a diameter of the Planck length. This model allows us to explain and calculate the intrinsic properties of the electron, such as its mass, charge, spin, etc., from the fundamental constants. Using this Planck sphere in four dimensions, we reach the conclusion that the electron particle has a size that is fixed by the Planck dimensions. The rotation of the Planck sphere generates the electron wave, the size of which depends on its wavelength. Our hypothesis is that the universe is composed of Planck spheres in four spatial dimensions, with two possible states: a rest state and rotational movement.
\end{abstract}

Keywords: Discrete Space-Time, Planck Length, Wave-Particle Duality, Electron Model

\section{Introduction}

Despite being the most thoroughly proven scientific theory, quantum mechanics $(\mathrm{QM})$ is difficult to interpret. Penrose (Penrose, 2004) lists up to six possible interpretations of the QM. For Einstein, the behaviour of the particles was determined individually, and he considered QM to be a probabilistic theory that was valid for use in studying the behaviour of sets of particles. The objective of this work is to propose a classical model of free electrons that agrees with the known experimental data and in which the individual behaviour of electrons is determined.

In 1923, Louis de Broglie suggested the possibility that particles of matter could have a "frequency of their own". This frequency was obtained by equating the Planck equation $(E=h v)$ to the Einstein energy $\left(E=m c^{2}\right)$ :

$$
v=\frac{m c^{2}}{h}
$$

This frequency can be observed experimentally in the Compton effect, and can be understood as a frequency of oscillation or rotation of the electron.

If the electron were spinning, it would generate both an electric current and a magnetic field, according to Maxwell's equations. The rotation of the electron would also generate an intrinsic magnetic moment.

"Ralph Kronig was the first to propose a spinning electron to explain the fine structure of atomic line spectra (in 1925), but he did not publish his results because there were too many problems with his idea. One of these problems was that the electron would have to rotate superluminall. Independently of Kronig, George Uhlenbeck and Samuel Goudsmit had the same idea." (Sebens, 2018).

If we assume that the size of the electron is the classical radius or Lorentz radius, to have an angular momentum of $1 / 2$, then the rotation speed of the electron must be superluminal (Tomonaga, 1997). Giffiths does this calculation and gets: $\mathrm{v}=5.15 \times 10^{10} \mathrm{~m} / \mathrm{s}$. “.... a point on the equator would be going more than 100 times the speed of light." (Griffiths, 2015)

Quantum mechanics evades the problem of the vibrational frequencies and superluminal rotation speeds of electrons by considering an electron to be a static particle without rotation. In this way, the properties of the electron such as its mass, charge, angular and magnetic momentum are intrinsic values of the electron, and are therefore not due to any type of real movement.

In addition to electrons, neutrinos are also considered punctual due to the way they appear in the standard model. However, quantum electrodynamics (QED) considers elementary particles to be points rather than point particles. 
"A point particle is the idealization of a real particle seen from so far away that scattering of other particles is as if the given particle were a point. Specifically, a relativistic charged particle is considered to be a point particle at the energies of interest if its interaction with an external electromagnetic field can be accurately described by the Dirac equation" (Neumaier, 2018).

For A. Burinskii "the nature of the electron is principal point for understanding of Quantum Theory" (Burinskii, 2012).

The Dirac electron model gives the value of the gyromagnetic factor as $g=2$. The same value was obtained by Carter in 1968 (Carter, 1968) from the Kerr-Newman solution, which initiated a series of works on the Kerr-Newman electron model (Debney, Kerr, \& Schild, 1969; Burinskii, 1972; Burinskii, 1974; Burinskii, 1994; Burinskii, 1995; Burinskii, 2002; Arcos \& Pereira, 2004; Burinskii, 2004, 2008 \& 2010).

The dispersion of high-energy electrons and positrons (Bourilkov, 2001) and high-precision atomic tests of QED (Odom, Hanneke, D’Urso, \& Gabrielse, 2006) show that the size of the electron is less than $10^{-20} \mathrm{~m}$.

Since 1915, various different models of the electron have been proposed. Parson (Parson, 1915) proposed that the electron was ring-shaped, and that the unit charge moved around the ring. This model was invalidated in 1923 by the Schrödinger wave equation.

Other researchers such as Jennison (Jennison, 1979) and Williamson and van der Mark (Williamson \& van der Mark, 1997) have proposed variations of the annular model that assume that an electron is a photon trapped in a vortex.

In 1930, Schrodinger found a solution to the Dirac equation that contained a term representing an oscillation of amplitude equal to the Compton wavelength. For Huang (Huang, 1952), this oscillation is the cause of the spin and the magnetic moment of the electron. Other authors such as Bunge (Bunge, 1995), Barut (Barut \& Zanghi, 1984) and Rivas (Rivas, 2001) have interpreted this oscillation as a real movement of the electron. In the helical electron model of Consa, the wavelength is proportional to the translation speed (Consa, 2018).

According Wilczek "There's no evidence that electrons have internal structure (and a lot of evidence against it)" (Wilczek, 2008). For Susskind, the electron radius is "most probably not much bigger and not much smaller than the Planck length" (Susskind, 2008) $\left(l_{p}=\left(G \hbar / c^{3}\right)^{1 / 2}=1.616 \cdot 10^{-35} \mathrm{~m}\right)$.

As early as 1925, Goudsmit and Uhlenbeck showed the electron had an intrinsic 'spin' that could be either clockwise or counterclockwise, and that the unfolding of the spectral lines of the atoms could be explained in this way (Uhlenbeck \& Goudsmit, 1925). This idea had previously been ruled out by other physicists, since it appeared that electrons would need to rotate faster than the speed of light; however, as we will see later, there are no superluminal velocities in the discrete electron model.

\section{Discrete Space-Time}

General relativity implies that space-time is a continuum. However, there is no experimental evidence for this. Are space and time a continuum or are they composed of indivisible discrete units? We're probably convinced of continuity as a result of education. In recent years however, both physicists and mathematicians have asked if it is possible that space and time are discrete? Smolin states that space is formed from atoms of space: "If we could probe to size scales that were small enough, would we see atoms of space, irreducible pieces of volume that cannot be broken into anything smaller?" that he calls "Atoms of Space and Time" (Smolin, 2004).

Minimum values of volume, length and area are measured in Planck units (Smolin, 2004). Planck's constant $h$, which represents the elementary quantum of action, has an important role in quantum mechanics.

Planck assumed that Newton's gravitational constant, Planck's constant and the speed of light were the most important universal constants. Using a dimensional analysis, he obtained the Planck mass, length, time and energy (Planck, 1899 \& 1906).

Motz considered that there was a fundamental particle called a uniton whose mass was equal to the Planck mass. The Planck particle would have irradiated most of its mass to become in the mass of subatomic particles (Motz, $1962 \& 1971)$.

De Has suggested that there were many Planck masses at the beginning of the universe, implying that the Planck mass has been reduced to the current values of the elementary particles (De, 2001).

Haug wrote that "Modern physics has also explored the concept of a hypothetical Planck particle that has $\sqrt{ } \pi$ more mass than the Uniton originally suggested by Motz"......." The Planck mass particle is, in our view, the mass-gap. It is a observational time-window dependent mass. We suspect that all other masses are time 
dependent as well, but this will first be noticeable when one is trying to measure their mass in a observational time window below their reduced Compton time, something we are not capable of doing at the moment" (Haug, 2018). The electron's mass can be found experimentally from the reduced Compton length of the electron (Prasannakumar, Krishnaveni, \& Umesh, 2012).

For some physicists, such as Hawking and Motz, Planck particles could be micro-black holes (Hawking, 1971; Motz \& Epstein, 1979; Obermair, 2013). It has also been suggested that the Planck mass is responsible for dark matter (Macgibbon, 1987; Dokuchaev \& Eroshenko, 2014).

Heisenberg showed that the mass of these particles must be derived from a fundamental length, together with the Planck constant $h$ and the speed of light (Heisenberg, $1943 \&$ 1957). The Planck scale combines gravity $(G)$, quantum mechanics $(h)$ and special relativity $(c)$ (Sprenger, Nicolini, \& Bleicher, 2012). According to Padmanabhan, the Planck length is the minimum length in any spacetime (Padmanabhan, 1985a, b).

Messen showed that the minimum length $a$ is given by the total energy of the universe $E_{u}$ in a four-dimensional space, $E_{u}=h c / 2 a$. The different excitations of space-time give rise to different particles (Meessen, 1999, 2011).

Haug proposed different methods of measuring the Planck length independently of the gravitational constant $G$. The Planck length is both a physical measurement and the diameter of the true fundamental particle: "The gravitational constant is a composite (derived) constant, while the Planck length represents something physical; it is the shortest reduced Compton wavelength possible. According to recent developments in mathematical atomism, there are also strong indications that the Planck length is the diameter of the only truly fundamental particle, namely an indivisible particle that together with void is making up all matter and energy" (Haug, 2017).

\section{4D Discrete Space}

The hypothesis is that the universe is composed of Planck spheres of four spatial dimensions, with two possible states: the rest state and rotational movement. Rest spheres are empty space, and the rotational motion of the spheres gives rise to different properties of the particles.

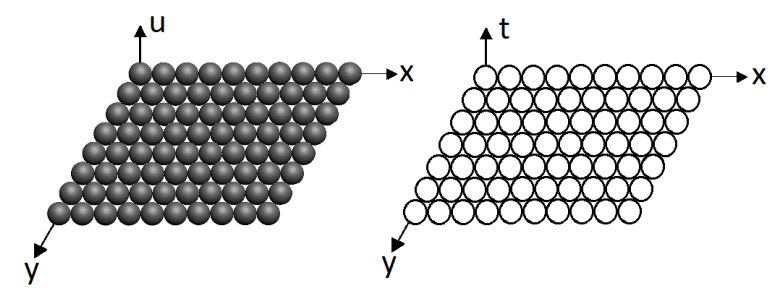

Figure 1. A 3D flat universe can be seen in terms of two spatial dimensions and time

Of the four dimensions, three are observed as space $(\mathrm{x}, \mathrm{y}, \mathrm{z})$ and the fourth $(u=c t)$ spatial dimension is observed as time. Planck's four-dimensional spheres are atoms of space and time that Smolin comments (Smolin, 2004). To simplify the drawing (Figure 1), only three dimensions are considered: $r(x, y)$ and $u$.

The hypothesis is that all particles are Planck spheres of four spatial dimensions (4D), whose diameter is the Planck length $l_{p}=\sqrt{G \hbar / c^{3}}$ (Figure 2). In addition the 4D Planck sphere has two rotations, one in three-dimensional space and one in the fourth dimension. Rotation in the fourth dimension $\left(\omega_{\mathrm{u}}\right)$ rotates the u-axis and another spatial axis around any two axes. For example, the $\mathrm{u}$ and $\mathrm{y}$ axes spinning around the $\mathrm{x}$ and $\mathrm{z}$ axes. In the rotation in space $\left(\omega_{\mathrm{e}}\right)$ it is rotated around the $\mathrm{u}$-axis and another spatial axis. For example, the $\mathrm{x}$ and $\mathrm{z}$ axes spinning around the $u$ and $y$ axes.

If space is made up of 4D Planck spheres, each sphere can only be at rest or spinning on its own axis. A 4D Planck sphere may rotate both in $3 \mathrm{D}$ space and in the fourth dimension $(u=c t$, Figure 2$)$, resulting in the following possible combinations (Garrigues-Baixauli, 2016; Baixauli, 2016; Garrigues-Baixauli, 2017a, b):

- zero rotations (vacuum space);

- one spatial rotation, $\omega_{e}$ (photons); 
- one rotation in the fourth dimension, $\omega_{u}$ (neutrinos);

- two rotations i.e. one spatial rotation, $\omega_{e}$, and one rotation in the fourth dimension, $\omega_{u}$ (first-generation electrons and quarks).

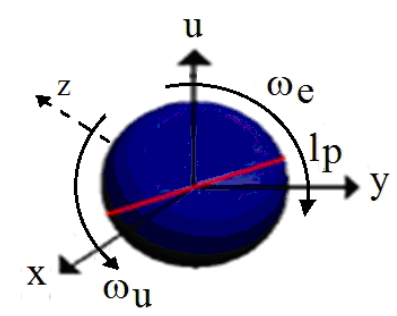

Figure 2. Rotations of a 4D Planck sphere

Static spatial spheres are not observed; it is what we call empty space. We can observe the spheres that rotate on themselves as elementary particles, such as electrons, photons and the first generation of quarks and neutrinos. The energy of rotation in the fourth dimension gives rise to the mass at rest and the period of rotation in the fourth dimension gives rise to the electric charge.

\section{Electron-Wave}

If we suppose that we have a particle of mass $\mathrm{m}$, which rotates at velocity $\omega_{e}$, the potential of the gravitational field at the distance $r$, will be:

$$
\frac{G m}{r}=v^{2}
$$

where $G$ is the gravitational constant, and v the velocity. Let us assume that this is the linear speed of rotation of the particle.The Planck sphere spin on itself one complete rotation in the three-dimensional space $\left(\omega_{\mathrm{e}}\right)$ in the same time as it turns in the fourth dimension $\left(\omega_{\mathrm{u}}\right)$, so that the particle is upside down $\left(\omega_{\mathrm{e}}=2 \omega_{\mathrm{u}}\right)$, what we see as $\operatorname{spin} 1 / 2$.

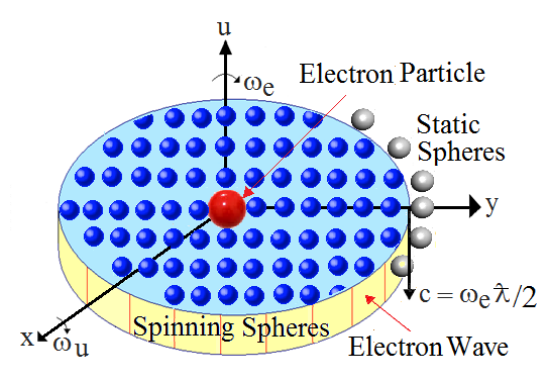

Figure 3. 2D representation of an electron

The spheres of space and time are linked by Planck's force, so that spinning one of them will drag it to adjacent spheres. The linear velocity of rotation (Figure 2) will increase as we move away from the rotating sphere, until the speed of light $\mathrm{c}$ is reached at a distance $\mathrm{r}$, then

$$
v=\omega_{u} l_{p}, c=\omega_{u} r
$$

The rest mass is due to rotation in the fourth dimension $\left(\omega_{t}\right)$, while a wave is due to a rotation in space $\left(\omega_{e}\right)$ or a disturbance in a medium. This medium is formed of the static 4D Planck spheres that constitute empty space.

Assuming that the potential of the gravitational field (Equation (2)) of a particle of mass $m$ is equal to the square of the linear speed of rotation $\left(v=\omega_{u} l_{p}\right)$ of a Planck sphere, as shown in Figure 4, we find that

$$
\frac{G m}{r}=v^{2}=\left(\omega_{u} l_{p}\right)^{2}
$$


Substituting $c=\omega_{u} r=\omega_{u} \lambda$ into Equation (4), and taking into account the Planck length, $l_{p}=\sqrt{G \hbar / c^{3}}$, we obtain

$$
E=m c^{2}=\frac{1}{2} \hbar \omega_{e}=\hbar \omega_{u}=\frac{\hbar c}{r}=\frac{\hbar c}{\hbar}
$$

where $\hbar$ is the reduced Planck constant, $r$ is the distance in the fourth dimension at which the adjacent space and time spheres rotate at the speed of light, and match the reduced Compton wavelength $\lambda=\lambda / 2 \pi$ of the electron. Therefore, the energy $\left(\hbar \omega_{u}\right)$ of the Planck sphere rotation in the fourth dimension $\left(\omega_{u}\right)$ is what we call mass, and the square of the linear speed of rotation of the Planck sphere is what we call potential of the gravitational field (Garrigues-Baixauli, 2016; Garrigues-Baixauli, 2017a, b).

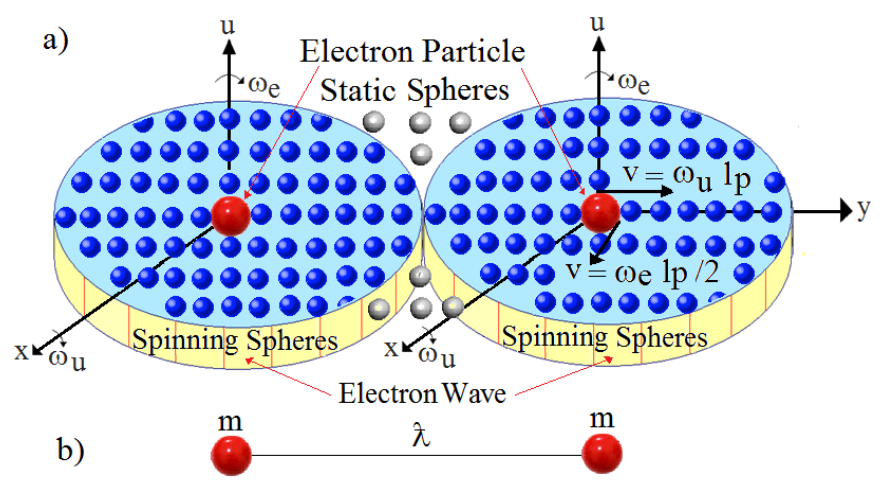

Figure 4. a) Discrete space; b) continuum space

\section{Relationship Between Mass and Electric Charge}

Let's suppose we have a circular loop carries a electric current I. Let's also say, that we reduce gradually the loop until we reach the size of Planck $\left(\mathrm{l}_{\mathrm{p}} \approx 10^{-35} \mathrm{~m}\right)$, and that the electric current flowing is equal to $1 \mathrm{~A}$. Under these conditions, the coulombs, which is the arbitrary units of electric charge, coincide with time in the seconds that the particle takes to spin.

In these conditions the period of the rotation in the fourth dimension gives rise to the electric charge. The mass will be due to the rotational energy of the Planck 4D sphere. This allows to relate the mass and the electric charge of the electron by applying the Heisenberg uncertainty principle (Garrigues-Baixauli, 2016; Baixauli, 2016; Garrigues-Baixauli, 2017a, b) as a principle of certainty.

$$
E=m c^{2}=\frac{1}{2} \hbar \omega_{e}=\hbar \omega_{u}=\frac{\hbar c}{r}=\frac{\hbar c}{\lambda}
$$

The mass $m$ is what we call the rest mass or inertial mass. Equation (5) can be expressed in terms of the period $\left(\omega_{u}=2 \pi / T_{u}\right)$, resulting in:

$$
E=m c^{2}=\frac{1}{2} \frac{h}{T_{e}}=\frac{h}{T_{u}}
$$

The electric charge will be due to the rotation $\omega_{u}$ (one of three possible rotations). Therefore, the electric charge can be expressed as

$$
q=\frac{1}{r^{2} c} \frac{\partial V_{4 D}}{\delta u}=2 \pi^{2} T_{u}
$$

Substituting $\left(T_{u}\right)$ into Equation (7), we obtain

$$
E=m c^{2}=\frac{2 \pi^{2} h}{q}
$$


where $m$ is the electron rest mass, and electric charge $q$ is in seconds.

$$
q=\frac{2 \pi^{2} h}{m c^{2}}=1.59710^{-19} s
$$

To preserve the units, it is only necessary to multiply Equation (12) by the unit of electric current $(I=1 \mathrm{~A})$. The rotation of the 4D Planck sphere explains the origin of the electric charge, rather than simply its description or measurement, as in current theories. According to Weinberg, "The aim of physics at its most fundamental level is not just to describe the world but to explain why it is the way it is" (Weinberg, 1993). The coulomb is an arbitrary unit of electric charge; current theory allows us to measure electric charge but not to explain it. An electric charge is simply defined as an intrinsic or fundamental property of matter (Garrigues-Baixauli, 2017a,b).

The electric charge can be seen as the period in the fourth dimension of the 4D Planck sphere, projected onto a 3D sphere.

The rotation of the 4D Planck sphere explains the origin of the electric charge, rather than simply a description or measurement of it as in current theories.

Being in the fourth dimension, mathematically electric charge can be expressed as imaginary $( \pm q i)$. Hence, the electrostatic force between charges of the same sign is repulsive, while the gravitational force is attractive. From our three-dimensional perspective, we can consider electric charge as real and distributed over the surface of Planck's 3D sphere.

The explanation given in this manuscript avoids superluminal velocities by assuming that the electron is a sphere whose diameter coincides with the Planck length. This is perfectly in accordance with measurements of the electric dipole moment of the electron. The electron appears to be spherical (Hudson et al., 2011; William et al., 2017; Baron et al., 2013) to within $1 \cdot 10^{-31} \mathrm{~m}$.

\section{Electron Particle Rest Mass}

Equation (5) can be expressed in terms of the Planck conditions, as follows:

$$
E_{p}=m_{p} c^{2}=\hbar \omega_{p}=\hbar c / l_{p}
$$

where $E_{p}$ is the Planck energy, $m_{\mathrm{p}}$ is the Planck mass, $\omega_{p}$ is the Planck rotation and $l_{p}$ is the Planck wavelength on $\hbar$.

Eliminating the Planck constant from Equations (5) and (11) gives

$$
m_{p} l_{p}=m \lambda
$$

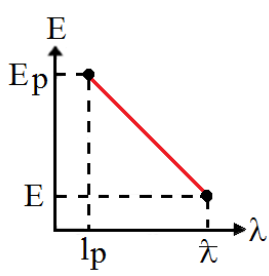

Figure 5. Energy as a function of wavelength

We now have an equation and two unknowns: the mass and wavelength, or the rotation and mass (Baixauli, 2016; Garrigues-Baixauli, 2017a). This indicates that the mass of the electron can vary from a minimum to a maximum value defined by the Planck mass.

We start from a Planck sphere with two rotations. The period gives rise to the electric charge, meaning that the electric force will initially be very small. Since the energy of rotation gives rise to the mass, the initial mass will 
be the Planck mass, and the initial gravitational force corresponds to the Planck force.

As the universe expands, the rotation of the 4D Planck sphere will decrease, resulting in a decrease in mass, an increase in the electric charge or rotation period (Garrigues-Baixauli, 2018) and therefore an increase in the electric force.

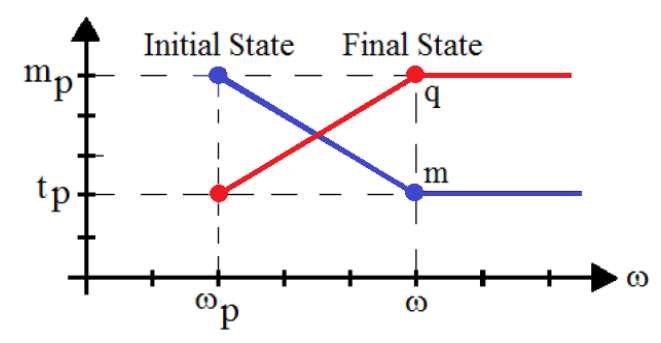

Figure 6. Initial and final states of the 4D Planck sphere

The 4D Planck sphere initially moves at the speed of light. As the rotation in the fourth dimension decreases, spheres in the adjacent 4D space enter and leave the 3D space that we observe. This decreases the speed of translation in space until the minimum speed is reached, i.e. when the electric force reaches its maximum value. We start from a gravitational force that is equal to the Planck force, meaning that the final state of the electric force should be the Planck force. This decrease in speed results in a decrease in electrical force compared to Planck force. In the final state, the electric force is:

$$
F_{E}=\alpha F_{p}
$$

i.e. when the electric charge of electron reaches the maximum value.

We can now write

$$
m \lambda c=m_{p} l_{p} c=\hbar
$$

Multiplying and dividing by the fine structure constant results in

$$
m \frac{\hbar}{\alpha} \alpha c=\hbar
$$

where $v=\alpha c$ is the velocity of the electron in a free state or with minimum energy, and which coincides with the speed of the electron in the hydrogen atom, according to the Bohr model. The electron is therefore a Planck sphere in four dimensions that is in a state of minimum energy. Equation (16) is the same as the Bohr atomic radius. If the initial moment is $\hbar$, the final moment will also be $\hbar$, and hence the electron in the hydrogen atom does not approach the nucleus. If the distance from the nucleus decreases, the speed increases, and vice versa.

On the other hand, when a particle of mass $\mathrm{m}$ and electric charge $q$ moving with velocity $\boldsymbol{v}$ penetrates a magnetic field $\boldsymbol{B}$, it describes a circular orbit whose radius is given by $q v B=m v^{2} / r$. But if the particle penetrates a gravitational field $\boldsymbol{g}$, the radius of the circular orbit is given by $m g=m v^{2} / r$. Equating both equations, it turns out, $q v B=m g$. If we do, $g=B=1$, then $m=q v$ (Baixauli, 2016).

In a universe formed by $4 \mathrm{D}$ Planck spheres, if the electric charge is the time that a Planck sphere takes to turn one spin in the fourth dimension, the rest mass must be space $(m=q v)$ and be related to the conditions of Planck. Under Planck conditions, it can be verified that

$$
K Q^{2}=G m_{p}^{2}
$$

where $K$ is the Coulomb constant, $G$ is the constant of gravitation, $Q$ is the Planck electrical charge and $m_{p}$ is the Planck mass. Thus, 


$$
m_{p}=\sqrt{\frac{K}{G}} Q=\sqrt{\frac{K}{G c^{2}}} Q c=\frac{1}{\sqrt{4 \pi}} \sqrt{\frac{\mu_{0}}{G}} Q c=\frac{1}{\sqrt{4 \pi}} \frac{Q c}{\alpha}
$$

where $\alpha$ is the fine structure constant. This also relates the constants of the gravitational and magnetic fields:

$$
G=\alpha^{2} \mu_{0}
$$

$$
6.6740810^{-11}=\left(7.297352566410^{-3}\right)^{2} \times 1.256637061410^{-6}=6.6917610^{-11}
$$

Obviously, in this equation the units do not coincide, because neither quantum mechanics nor general relativity explain the origin of the rest mass or the electric charge. These theories only assign arbitrary units to them and consider them independent intrinsic properties. If it is intended to recover the typical units of these parameters, the equation (19) can be simply multiplied as follows

$$
G J^{2}=\alpha^{2} \mu_{0} I^{2}
$$

Where $J$ is the linear mass density $\left(\mathrm{J}=1 \mathrm{~kg} \mathrm{~m}^{-1}\right)$ and $I$ is the electric current unit $(\mathrm{I}=1 \mathrm{~A})$.

Taking into account Equations (15) and (18), we can write

$$
m_{p} l_{p}=\frac{1}{\sqrt{4 \pi}} \frac{Q c}{\alpha} l_{p}=m \lambda
$$

Where

$$
m=\frac{1}{\sqrt{4 \pi}} \frac{Q_{c}}{\alpha} \frac{l_{p}}{\lambda}=\frac{q c}{\sqrt{4 \pi \alpha}} \frac{l_{p}}{\lambda}
$$

From Equations (5) and (9), the wavelength of the electron can be expressed in terms of the electric charge, as follows

$$
\lambda=\frac{q c}{2 \pi^{2}}=2 \pi \lambda
$$

Substituting into Equation (22), we obtain the mass of the electron in metres.

$$
m=2 \pi^{2} \sqrt{\frac{\pi}{\alpha}} \frac{l_{p}}{\alpha}=9.08610^{-31} m
$$

The mass is the space in the fourth dimension of the 4D Planck sphere, projected onto the 3D sphere that we observe as a particle.

In a universe made only of space and rotation of 4D Planck spheres, if the electric charge is time, the rest mass can only be space.

Paul Dirac in 1963 wrote: "The physics of the future, of course, cannot have the three quantities $h$ e and c all as fundamental quantities. Only two of them can be fundamental, and the third must be derived from those two. It is almost certain that $c$ will be one of the two fundamental ones. The velocity of light, $c$, is so important in the four-dimensional picture, and it plays such a fundamental role in the special theory of relativity, correlating our units of space and time, that it has to be fundamental (Dirac, 1963).

In fact, the electric charge has been obtained as a function of $\mathrm{h}$ and $\mathrm{c}$, since $\mathrm{c}$ and $l_{p}$ determine the initial conditions of the 4D Planck sphere.

\section{Intrinsic Angular Momentum or Spin}

The rotation $\omega_{u}$ in the fourth dimension of a 4D Planck sphere causes the intrinsic angular momentum or spin of the electron. 


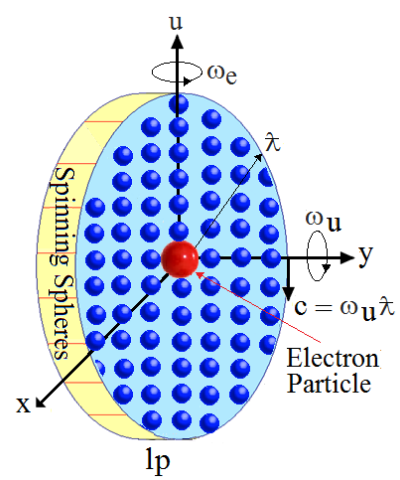

Figure 7. Spin of an electron

The intrinsic angular momentum is

$$
L=I \omega_{u}=\frac{1}{2} m r^{2} \omega_{u}=\frac{1}{2} m \hbar c=\frac{1}{2} \hbar
$$

Figure 8. Positive and negative electron spin

The rotation can be clockwise or counterclockwise; for example, a clockwise rotation may be spin $+1 / 2$ and a counterclockwise rotation may be $-1 / 2$.

Since the electron can rotate in either direction, there is no way of knowing which direction a given electron will take. The sign of the spin cannot therefore be known without observing it, since it is equally likely to be positive or negative. Instead, it is possible to select two particles in such a way that the sum of their angular momenta is zero. Although one particle will rotate in one direction and the other in the opposite direction, it is necessary to measure the state or rotation of one in order to determine that of the other.

The rotation in the fourth dimension exerts a drag on the spheres in 4D space, decreasing the speed of the electron in $3 \mathrm{D}$ space.

\section{The Quantum Tunnelling}

At a figure skating exhibition, we observe that when the skaters approach their arms to their body their spinning velocity $\omega$ increases and the inertial moment I decreases, which demonstrates the conservation of angular moment law.

On the other hand, the rotational kinetic energy is greater when the rotational velocity is greater. This energy increase is produced at the expense of a decrease of the internal potential energy then the mechanic energy does not change.

Let's suppose that we cannot see the skater but we can measure his rotational kinetic energy. The skater is at all times with his arms outstretched, unless there is an obstacle. After running some measurements from the grandstand, but varying the distance $\left(x_{1}, x_{2}, x_{3}\right)$ to which we run the measurement, we reach to the "erroneously conclude" that the rotational kinetic energy $\left(E_{m}\right.$, Figure 9$)$ is a skater's intrinsic property. A skater that we cannot see. Let's also suppose that as the technology improves we get closer to the skater. In order to keep on spinning the skater get his arms closer to his body, in a way that when we measure the energy at distance lower than the length of his arms we observe how the E' energy has increased. The maximum energy will be $E_{M}$, when the skater has his arms against his body. In the graphic $L$ represents the length of the skater's arms. 


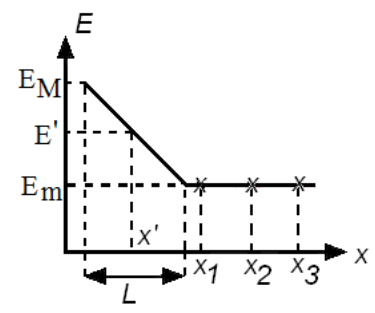

Figure 9. Skater's rotational kinetic energy according to the distance measured

Both the quantum mechanics and the classical or relativistic physics suppose that mass of the particle is an intrinsic property of the matter which is independent from the measurement state. The equation (12) indicates that the mass of the particle is not defined except for the minimum energy state. For instance, the electron mass can be any value between what we call rest mass and Planck's mass (Figure 5).

From the hypothesis that the universe and the particles are formed by four dimension space-time spheres, it can be deduced that the particles are Planck spheres in a minimum energy state. This energy is due to the four dimensions Planck's sphere rotation. This spin drags the adjacent space-time spheres to a distance equal to half of its wavelength $\lambda$ at rest (Equation 5).

If the particle is far from the potential barrier in a way that the distance is equal or greater than its wavelength (Figure 10a), then its energy is lower than the potential barrier one.

As the particle approaches the potential barrier, just like the skater withdraws his arms, the particle increases its rotation (Figure 10b) and so its energy increases, until its energy reaches the value $V_{o}$ (Figure 10c) and overcomes the barrier. Once the barrier has been overcome, the particle returns to the lowest energy state (Figure $10 \mathrm{~d})$.
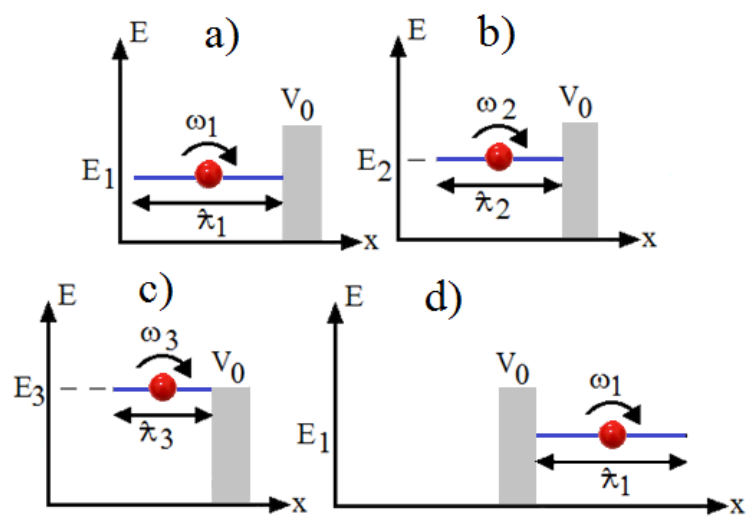

Figure 10. Classical quantum tunneling

Any perturbation makes the particle vary its energy keeping the angular momentum. Once the obstacle has been overcome the particle returns quickly to the lowest energy state.

The problem lies in supposing that the mass of the particle is an intrinsic property of the matter, since the current theories (quantum mechanics and general relativity) does not allow to explain what the mass is.

The current universe consists of different particles, energy, and space-time vacuum; all these elements originated from the energy of the Big Bang. It is assumed that they were initially identical and remain identical. As Einstein believed, electron is simultaneously a particle and a wave.

There is not probability of finding a particle on the other side of the barrier. The wave function has nothing to do with the probability. The wave function is the ratio between the kinetic energy that the electron has when it is unobserved and the energy that it acquires due the observation (Garrigues-Baixauli, 2016; Garrigues-Baixauli, 2017a). Simply, the energy of the particle increases momentarily, in the same way as the skater's energy increases, and is capable of overcoming the barrier if he gets close enough. At all moments the particle energy 
verifies the equality in the Heisenberg's uncertainty principle, as a principle of certainty.

\section{Special Relativity}

\subsection{Relativistic Mass}

The rotation $\omega_{e}$ in the space of the Planck 4D sphere determines the speed of translation of the particle in 3D space; the greater the energy, the greater the speed.

Since the rotation in space $\omega_{e}$ is perpendicular to the rotation in the fourth dimension $\omega_{u}$, its energies will also be perpendicular. The total energy of the particle will therefore be the vector sum of both energies.

$$
\vec{E}_{t}=\vec{E}_{r}+\vec{E}_{u}
$$

where $E_{t}$ is the total energy of the particles, $E_{r}$ is the energy due at the momentum $p=m v$, and $E_{u}$ is the energy at rest

$$
E_{u}=\hbar \omega_{u}=m_{0} c^{2}
$$

where $m_{0}$ is the mass at rest. For small or non-relativistic speeds $(\mathrm{v}<<\mathrm{c})$, we have:

$$
E_{r}=\frac{1}{2} \hbar \omega_{e}=\frac{1}{2} m_{0} v^{2}
$$

However, for relativistic speeds close to the speed of light, we must also take into account the intrinsic angular momentum or rotation of the Planck sphere in 3D space.

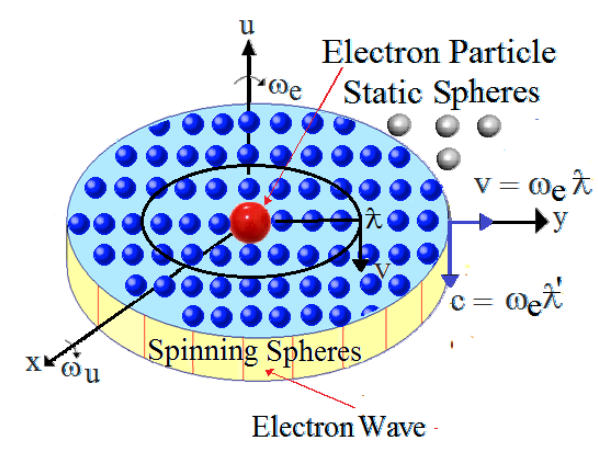

Figure 11. Relativistic electron

The angular momentum will be

$$
L=I \omega_{e}=\frac{1}{2} m \hbar^{2} \omega_{e}=\frac{1}{2} m \lambda v=\frac{1}{2} \hbar
$$

where $v=\omega_{e} \lambda / 2 \pi$ is the speed of displacement in 3D space, and the wavelength $\lambda$, is the distance that the Planck particle travels while to rotate a complete round. Rotation at a speed $v$ generates a wave with wavelength

$$
\lambda=\frac{h}{m v}
$$

Where $\_$is the de Broglie wavelength. This wavelength $\lambda$ is the distance that the electron particle travels during one rotation at an angular velocity of $\omega_{e}$. As it spins, the electron particle exerts drag on the adjacent 4D Planck spheres, forming the electron wave. As 4D Planck spheres move away from the particle electron, the linear speed of rotation will increase until it reaches the speed of light $\mathrm{c}=\omega_{\mathrm{e}} \lambda^{\prime} / 2 \pi$. Then, the distance $\lambda^{\prime} / 2 \pi$ is the radius of the flat disc formed by $4 \mathrm{D}$ Planck spheres that rotate with the electron particle and that constitute the wave associated with the electron particle that is observed. Hence, $\lambda^{\prime} / 2 \pi$ is the maximum amplitude of the wave.

According to de Broglie, the movement of a particle is governed by the propagation of the pilot wave associated with it. 
As the energy of the particle increases, so does the rotation speed $\omega_{e}$. Consequently the mass of the particle increases and its radius decreases, meaning that the angular momentum is conserved.

The particle does not know that there is an angular momentum, it does not know what energy is, it also does not know the principle of conservation of angular momentum. As its rotation increases, this translates into an increase in what we call mass (Equation (22)) or rotational energy (Equation (6)). There is also a decrease in the radius $\lambda$ that gives rise to the principle of conservation of angular momentum. When the radius is reduced, the period decreases or an equivalent temporary dilation is generated due to the increase in the speed.

Equation (30) can be expressed as

$$
m v c=\frac{\hbar c}{\lambda}
$$

Hence, the total energy will be

$$
E_{t}=c \sqrt{(m v)^{2}+\left(m_{0} c\right)^{2}}
$$

The above equation is the energy-momentum relation

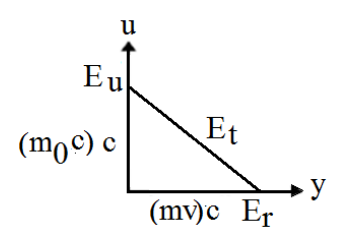

Figure 12. Particle energies

At small speeds, $m$ and $m_{0}$ coincide, but at relativistic speeds, the mass $m$ increases due to the increase in the rotation energy $E_{r}$. If $m v<<m_{o} c$, we can approximate the total energy to the kinetic energy at a velocity $c$. Then,

$$
\begin{gathered}
m c^{2}=c \sqrt{(m v)^{2}+\left(m_{0} c\right)^{2}} \\
m^{2}\left(c^{2}-v^{2}\right)=m_{0}^{2} c^{2} \\
m=\frac{m_{0}}{\sqrt{1-v^{2} / c^{2}}}
\end{gathered}
$$

The electron is a Planck sphere in the minimum energy state $\left(m_{0}\right)$. Hence, the maximum energy will be the Planck energy $\left(m_{p} c^{2}\right)$, and

$$
v=c \sqrt{1-\frac{m_{0}^{2}}{m_{p}^{2}}}
$$

Taking into account Equation (12), we obtain

$$
v=c \sqrt{1-\frac{l_{p}^{2}}{\lambda^{2}}}
$$

where $l_{p}$ is the Planck length and $\lambda$ is the reduced Compton wavelength of the electron.

This gives us a maximum speed for the electron.

Haug (Haug, 2014, 2016, 2017a, b) has suggested that there is a maximum velocity for any particle with a rest mass, as given by the previous equation. Haug has also calculated this maximum speed. "For any observed 
particle, the maximum velocity will be very close to that of the speed of light, but considerably above the speed achieved in the Large Hadron Collider. An electron has a reduced Compton wavelength of $\lambda_{e} \approx 3.8165910^{-13} \mathrm{~m}$ and here we suggest that it can never be accelerated to a velocity faster than

$$
\mathrm{v}=\mathrm{c} \times 0.99999999999999999999999999999999999999999999912416
$$

In the above calculation, we have assumed a Planck length of $1.61619910^{-35} \mathrm{~m}$ " (Haug, 2018).

4D Planck spheres are always spheres regardless of the movement of the observer. In Equation (37) appears the Planck length, which is the minimum size that the electron can have. When the energy is applied to the electron, its rotation increases and therefore the size of the flat disk decreases $\left(n l_{p}\right)$. What varies is n, not the diameter $l p$ of the $4 \mathrm{D}$ spheres. When energy is applied to an object, the spatial rotation $\left(h \omega_{e}\right.$, Equation (27)) and the angular momentum change, but not the rotation in the fourth dimension $\omega_{\iota}$. Therefore the rest mass does not change and the electric charge does not either.

The macroscopic spheres cease to be spheres for an observer moving in a continuous space-time. In a discrete space of 4 spatial dimensions that we observe as discrete space-time there is no contraction of the minimum discrete space, but the space occupied by the object varies, $1=\mathrm{n} l \mathrm{p}, \mathrm{n}$ decreases with speed.

At the current energy scale, the spacetime can be considered as continuous, but it is not correct to put $l=0$, because then it is no longer discrete. Thus infinities and singularities disappear. The evolution of the particle does not change because it is considered finite, almost punctual instead of punctual.

Therefore, relativistic effects are due to the contraction of physical objects as they move through 4D space. 4D space can be considered a fundamental frame of reference or "ether" according to the alternative interpretation of the special relativity of Lorentz (Lorentz, 1904), Poincaré (Poincaré, 1905, 1906) and others (Builder, 1958; Prokhovnik, 1967).

"The "relativistic" effects, which essentially follow from the Lorentz transformation, are all due to the "true" Lorentz contraction of physical objects as they are moving through the "ether" or fundamental inertial frame" (Arminjon, n. d.).

On the other hand, the rotation $\omega_{u}$ defines the intrinsic properties of the electron, such as: mass, electric charge, spin, etc. Therefore, there is no need to change the angular velocity $\omega_{u}$ between different observers, since these properties do not change between observers. In contrast, the angular velocity $\omega_{e}$ defines the linear momentum and this varies with the velocity. Therefore, the angular velocity $\omega_{e}$, varies in the same way that the mass varies between different observers.

Multiplying equation (35) by the square of the speed of light, results

$$
E=m c^{2}=\frac{m_{0} c^{2}}{\sqrt{1-v^{2} / c^{2}}}
$$

And taking into account the equation (5)

$$
E=\hbar \omega_{e}=\frac{\hbar \omega_{e 0}}{\sqrt{1-v^{2} / c^{2}}}
$$

\subsection{Dilation of Time}

When we apply energy to the electron, its wavelength decreases. In the same way, when applying energy to an atom, the distances of the different energy levels decrease and therefore the frequency of each transition increase.

On the other hand, temporary dilation has been proven in atomic clocks. Therefore, by decreasing the period of the transition, the time for the same number of transitions will decrease. Hence, the atomic clock in motion indicates a shorter time than the atomic clock at rest.

The equation (40), can be put in function of the period of rotation 


$$
E=\hbar \frac{2 \pi}{t^{\prime}}=\frac{\hbar 2 \pi / t}{\sqrt{1-v^{2} / c^{2}}}
$$

Where $t^{\prime}$ is the period of rotation of the particle in motion and the period of rotation of the particle at rest

$$
t^{\prime}=t \sqrt{1-\frac{v^{2}}{c^{2}}}
$$

We need the concept of inertial system to be able to know which object moves (twin paradox). However, the particles know perfectly what particle is moving, because their energy in space $\left(\hbar \omega_{e}\right)$ is greater than their energy at rest $\left(\hbar \omega_{e 0}\right)$. Therefore the period $t$, which corresponds to the rotation $\omega_{\mathrm{u}}$ does not vary with the speed. What varies is the time that the particle moves in space (Figure 13).

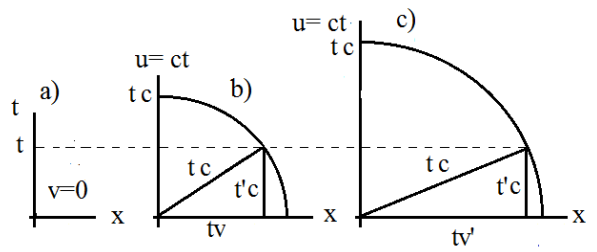

Figure 13. a) observer at rest. b) observer in motion at speed v. c) observer in motion at the speed v'> v

$t$ is the time that the observer (object) at rest moves at the speed $\mathrm{c}$ in the fourth dimension. $t^{\prime}$ is the time that the observer (object) moves in the fourth dimension at the velocity $v_{u}=t^{\prime} / t c$, while moving in space at the velocity $v$. Therefore, it is a movement in the $\mathrm{x}-\mathrm{u}$ plane, so it is not correct to calculate the distance traveled by the observer in motion as t'v, except for small speeds. The observer at rest moves only in the fourth dimension. at speed c, while the moving observer moves in the plane $\mathrm{u}-\mathrm{r}(\mathrm{x}, \mathrm{y}, \mathrm{z})$ at the velocity c. Therefore, at all times we move at the speed of light. When applying energy to an object, what changes is the direction of movement.

\section{Magnetic Moment}

The magnetic moment of the electron is another intrinsic property of matter. If the electron is a point with electric charge in repose, it should not have magnetic moment as the moment is due to the movement of the mass (angular momentum) or the electric charge (magnetic moment).

We consider a particle of mass $m$, with velocity $v$, moving under the influence of a force $F$. The angular momentum modulus in a particle about point $\mathrm{O}$ of the space is defined as the product of his linear momentum $(m v)$ multiplied by the distance $(r)$ relative to that point.

Applying the definition of angular momentum we can define the modulus of the magnetic moment of a particle about point $\mathrm{O}$ of the space as the product of its charge in movement ( $q v)$ multiplied by the distance $(r)$ relative to that point.

On the other hand, as for the hydrogen atom, the electron spins in a radius and velocity spherical orbit

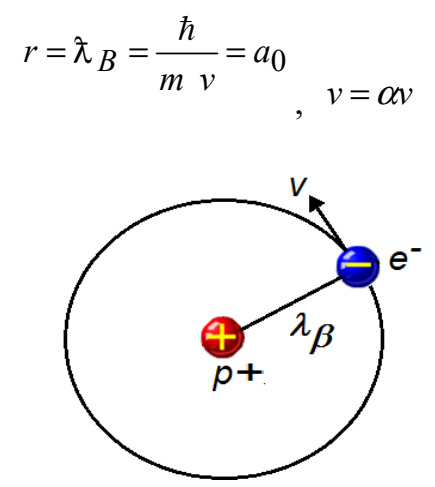

Figure 14. Bohr atomic model 
Therefore, applying the definition of angular momentum results in

$$
\mu_{B}=\frac{q}{2} v r=\frac{q}{2} v \frac{\hbar}{m v}=\frac{q \hbar}{2 m}
$$

It is the Bohr magneton and where $q$ is the electron charge, $\hbar$ is the Planck's reduced constant and $m_{e}$ the of the electron rest mass.

Since the electron is a charged particle, the electron spin must give rise to a magnetic moment $\boldsymbol{\mu}$ intrinsic or spin's. The relation between the magnetic moment vector and the spin is

$$
\mu=\frac{1}{2} g \frac{q \hbar}{2 m_{e}}=\frac{1}{2} g \mu_{B}
$$

Where $g$ is the spin magnetic reason of the electron, its experimental value is (Mohr, Taylor, \& Newell, 2016)

$$
2.00231930436182
$$

The predicted value by the Dirac theory is 2 , showing a slight divergence with the measured value. If the electron were a one-off particle as the QM proposes, the magnetic moment of the experimentally measured electron should be the predicted value by the Bohr magneton.

In the model detailed in this monograph, the electron has and internal structure formed by the 4 dimensions Planck atoms, which spin respectively in the three spatial dimensions as well as in the fourth dimension. Thus, such structure will be taken into account when calculating the magnetic moment.

Let's see how such structure influences.

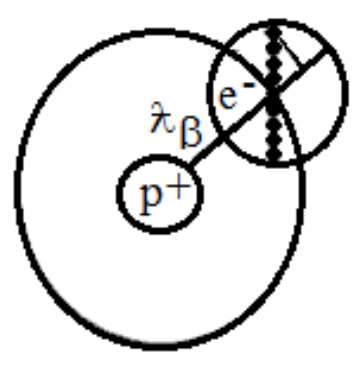

Figure 15. Hydrogen atom atomic model. Electron with structure

The electron spins around the proton at a $\lambda_{B}$, distance, in addition, we must take into consideration its radius projection $\lambda / 2$, while it spins around its center. Therefore, the distance $r$, to the center of the proton will be

$$
r=\lambda_{B}+r^{\prime}=\lambda_{B}+\frac{\lambda / 2}{2 \pi}=\lambda_{B}+\frac{\alpha \lambda_{B}}{4 \pi}=\lambda_{B}\left(1+\frac{\alpha}{4 \pi}\right)
$$

Additionally, meanwhile the electron takes a spin in the space, it takes half spin in the fourth dimension, hence we have the electron upside down (1/2 spin).

Thus, we must consider half of the electron's electric charge, resulting in a magnetic moment

$$
\mu_{B}=\frac{q}{2} v r=\frac{q}{2} \omega r^{2}
$$

With $v=\omega r$, being $\omega$ the angular speed of rotation. Squaring equation (46) and substituting in equation (47) results in

$$
\mu_{B}=\frac{q}{2} \omega \lambda_{B}^{2}\left(1+\frac{\alpha}{4 \pi}\right)^{2}
$$


And taking into account equation (39), results in

$$
\mu_{B}=\frac{q \hbar}{2 m}\left(1+\frac{\alpha}{4 \pi}\right)^{2}
$$

Finally, taking the equation into account, the gyromagnetic factor will be

$$
g_{e}=2\left(1+\frac{\alpha}{4 \pi}\right)^{2}=2\left(1+\frac{\alpha}{2 \pi}+\frac{\alpha^{2}}{16 \pi^{2}}\right)=2.002323494
$$

The first term in $\alpha$, matches with the cone calculated by Schwinger. The gyromagnetic factor matches with an accuracy of 5 significant numbers with the value experimentally measured.

\section{Electrons and Positrons}

In 1928, Dirac predicted that each fundamental particle has a corresponding antiparticle. Years later, the positron or antiparticle of the electron was discovered. In 1937, Majorana predicted that fermions would be found that were their own antiparticles. In 2017, a team of physicists found solid-state particles that are similar to these Majorana fermions.

According to the authors "Although Majorana fermions remain elusive as elementary particles, their solid-state analogs have been observed in hybrid semiconductor-superconductor nanowires. In a nanowire setting, the Majorana states are localized at the ends of the wire" (He et al., 2017).

Wheeler and Feynman showed that electrons and positrons are the same particle; electrons move into the future and positrons into the past. "I took the observation that positrons could simply be represented as electrons going from the future to the past in a back section of their world lines" (Feynman, 1965). "In view of the fact that in classical physics positrons could be viewed as electrons proceeding along world lines toward the past" (Feynman, 1949).

Planck's 4D sphere can rotate in three-dimensional space $\left(\omega_{\mathrm{e}}\right)$ or in fourth dimension $\left(\omega_{\mathrm{u}}\right)$. If we change the direction of rotation of the spatial rotation the positive particle becomes negative.

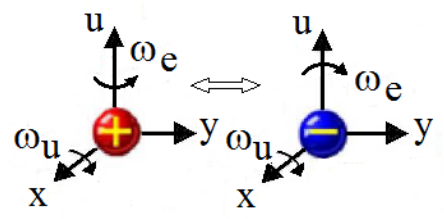

Figure 16. The electron and the positron are the same particle

Our spatial perspective lets us see both directions of a given physical dimension at the same time, for example both up and down. The fourth spatial dimension is observed as time; however, our temporal limitation prevents us from observing the past and the future simultaneously. Therefore, from our temporal perspective, these are two different particles. We also need a sign (+ or - ) to distinguish these in mathematical or physical equations.

\section{Quantum Entanglement}

Although local realism forms the basis of classical physics and general relativity, quantum entanglement is a feature of quantum mechanics that rejects this principle.

Quantum entanglement is one of the most disconcerting phenomena of quantum mechanics. When two particles such as electrons, atoms or photons are intertwined, there is an inexplicable link between them that remains even if the particles are separated. These intertwined particles form a single system, with a common wave function for both particles, and cannot be defined using a wave function for each individual particle. Quantum entanglement implies that any measurement made on the first particle provides information about the result of measuring the second particle.

According to QM, the properties of the particles are not defined before they are observed, and the particles take these properties only when they are observed. These properties are not programmed in advance, but are defined 
at random. We cannot therefore predict the outcome of a measurement, and can only give the probability of something happening. In an entangled state, when one of the particles is manipulated, the state of the other is modified instantaneously, even if the particles are very far apart from each other. This implies that there is a correlation between the particles that does not take place in classical physics.

The spins of two electrons can be interlinked so that the spin of each electron depends on that of the other, although each separate spin is unknown. Indeed, it has been shown previously that the spin of each electron corresponds to an intrinsic angular momentum in the classical sense. Any rotation may be clockwise or counterclockwise, and there is therefore no way to know the direction of rotation unless it is measured. To distinguish one rotation from another in a mathematical sense, it is necessary to include the sign, + or - . Half of any set of electrons will have $+1 / 2 \hbar$ (spin up) and the other half $-1 / 2 \hbar$ (spin down). Thus, if we send a pair of electrons to a pair of observers, i.e. one to Alice (observer A) and another to Bob (observer B), there is no way to know the spin seen by each observer, meaning that in principle, we have four combinations.

The spins of a pair of electrons can be combined to form a state of spin zero, so that when a particle is observed to have spin $+1 / 2$, the other will automatically have spin $-1 / 2$. Entanglement is equivalent to sending one shoe from a pair (i.e. a left shoe and a right shoe) to each observer; hence, entanglement reduces the number of different combinations by half, meaning that there are only two possible combinations in this case. The independent classical probability therefore becomes a conditioned or dependent probability.

Entanglement is only possible for a pair of particles with zero total spin, and this therefore eliminates combinations of the same sign (++ and --). The wave function used is therefore common to combinations of different signs $(+-$ and -+$)$.

Rotation in the fourth dimension $\left(\omega_{u}\right)$ gives rise to the spin of the electron. The electron also has rotation in 3D space. After a certain time $T_{e}$, the clockwise rotation becomes counterclockwise and vice versa, since the rotation in space causes us to 'see' the reverse of the particle (if it makes sense to speak of the front and back of a sphere) The spin of the electron is therefore undefined until it is measured. As quantum mechanics affirms, we can consider that each electron has both spins, but not simultaneously. In other words, the spin of the electron changes over time, but synchronously with the other entangled particle.

In short, the probability of the spin seen by Bob is conditioned by the probability of the spin seen by Alice. Bell did not take into account a probability conditioned on this inequality.

Fisher et al, say "We show that paradoxical consequences of violations of Bell's inequality are induced by the use of an unsuitable probabilistic description for the EPR-Bohm-Bell experiment. The conventional description (due to Bell) is based on a combination of statistical data collected for different settings of polarization beam splitters (PBSs). In fact, such data consists of some conditional probabilities which only partially define a probability space. Ignoring this conditioning leads to apparent contradictions in the classical probabilistic model (due to Kolmogorov). We show how to make a completely consistent probabilistic model by taking into account the probabilities of selecting the settings of the PBSs. Our model matches both the experimental data and is consistent with classical probability theory" (Fischer, Hilbert, \& Khrennikov, 2008).

Also for A Khrennikov, quantum entanglement is a classical probability of probability interference. "We demonstrate that the EPR-Bohm probabilities can be easily obtained in the classical (but contextual) probabilistic framework by using the formula of interference of probabilities. From this point of view the EPR-Bell experiment is just an experiment on interference of probabilities. We analyze the time structure of contextuality in the EPR-Bohm experiment. The conclusion is that quantum mechanics does not contradict to a local realistic model in which probabilities are calculated as averages over conditionings/measurements for pairs of instances of time $t 1<t 2$ " (Khrennikov, 2004).

\section{Conclusion}

The electron does not know that there is a principle of conservation of energy, nor does it know the principle of conservation of angular momentum, let alone know the value of the Planck constant. The electron is simply a 4D Planck sphere with two rotations. The period in the fourth dimension creates what we call electric charge, while the speed of rotation in space multiplied by the period gives the mass. We can also consider mass as the energy of rotation. From our perspective, when we apply energy, for example in a particle accelerator, the electron simply increases its rotation, which results in an increase in the energy, speed of translation and mass. As a further consequence of this increase in the speed of rotation $\left(c=\omega_{e} r\right)$ or spin, there is a decrease in the radius of the flat disc that constitutes the electron wave. The electron particle maintains its size. The same effect arises when we use photons to measure the position or momentum of the electron, and the electron increases its 
rotation.

Regardless of whether this energy comes from a particle accelerator or a photon, the rotation of the electron increases. Physicists simply measure something and obtain a number; this number is given a name and is assigned a unit of measurement. We then look for an equation that matches the measured values, and can also predict new values to verify that the equation is correct. However, we are not concerned with explaining what this measure produces. This means that current theories are becoming increasingly complex and need larger numbers of constants. In addition to describing what is observed, some scientists maintain that physics should explain why nature is as it is. This paper presents a model that tries to explain the nature of QM.

The electron and photon are simply 4D Planck spheres with two rotations. The different speeds of these rotations cause the different properties that are observed. From our point of view, different constants are needed to explain different properties; for example, a mass can be converted into a frequency through the concept of energy. The only equation that governs the behaviour of the electron is $c=\omega r$, and the other equations are simply needed to convert these rotations into the concepts, values and units used in physics.

\section{Conflict of interests}

The authors declare that there is no conflict of interests regarding the publication of this paper.

\section{References}

Arcos, H. I., \& Pereira, J. G. (2004). Kerr-Newman solution as a Dirac particle Gen. Rel. Grav., 36, 2441.

Arminjon, M. (n. d.). Gravitational Energy Loss and Binary Pulsars in the Scalar Ether-Theory of Gravitation.

Baixauli, J. G. (2016). Relation Between the Gravitational and Magnetic Fields. J. Phys. Math., 7, 169.

Baron et al. (2013). Order of Magnitude Smaller Limit on the Electric Dipole Moment of the Electron.

Barut, A. O., \& Zanghi, N. (1984). Classical Model of the Dirac Electron. Phys. Rev. Lett., 52, 2009.

Bourilkov, D. (2001). Hint for axial-vector contact interactions in the data on $\mathrm{e}+\mathrm{e}-\rightarrow \mathrm{e}+\mathrm{e}-(\gamma)$ at center-of-mass energies 192-208 GeV. Phys. Rev. D, 64, 071701. Physical Review Online Archive.

Builder, G. (1958). Austr. J. Phys., 11, $279 \& 457$.

Bunge, M. (1995). A picture of the electron. Nuovo Cimento, 1, 977.

Burinskii, A. (1972). Microgeon with Kerr metric Abstracts of the III Soviet Gravitational Conference (p. 217).

Burinskii, A. (1974). Microgeons with spins Sov. Phys. JETP, 39, 193.

Burinskii, A. (1994). String-like structures in complex Kerr geometry. Journal of Physics: Conference Series, 532 , 1.

Burinskii, A. (1995). Some properties of the Kerr solution to low-energy string theory. Phys. Rev. D, 52, 5826.

Burinskii, A. (2002). Supersymmetric Superconducting Bag as a Core of Kerr Spinning Particle Grav. Cosmol., 8 , 261.

Burinskii, A. (2004). Twistorial analyticity and three stringy systems of the Kerr spinning particle. Phys. Rev. D, 70.

Burinskii, A. (2008). The Dirac-Kerr-Newman electron. Grav. Cosmol., 14, 109.

Burinskii, A. (2010). Regularized Kerr-Newman solution as a gravitating soliton. J. Phys. A: Math. Theor., 43, 392001.

Burinskii, A. (2012). Gravity versus Quantum Theory: Is electron really pointlike? J. Phys. Conf. Ser., 343.

Carter, B. (1968). Global structure of the Kerr family of gravitational fields. Phys. Rev., 174, 1559.

Consa, O. (2018). Helical Solenoid Model of the Electron. Prog. in Phys., 14(2).

De, S. S. (2001). Quantum creation of highly massive particles in the very early universe. International Journal of Theoretical Physics.

Debney, G. C., Kerr, R. P., \& Schild, A. (1969). Solutions of the Einstein and Einstein-Maxwell equations. J. Math. Phys., 10, 1842.

Dirac, P. (1963). The Evolution of the Physicist's Picture of Nature. Scientific American, 208(5), 45-53.

Dokuchaev, V. I., \& Eroshenko, Y. N. (2014). Black hole atom as a dark matter particle candidate.

Feynman, R. (1949). The Theory of Positrons. Phys Rev, 76, 749. 
Feynman, R. (1965). The Development of the Space-Time View of Quantum Electrodynamics. Nobel Lecture. Nobel Foundation.

Fischer, P., Hilbert, A., \& Khrennikov, A. (2008). Complete account of randomness in the EPR-Bohm-Bell experiment.

Garrigues-Baixauli, J. (2016). Derivation of the Schrodinger Equation from Classical Physics. Int. J. Sci. Res., 5, 77-80.

Garrigues-Baixauli, J. (2017a). Classic 4D Model of the Discrete Universe. I: Quantum. Scholars' Press.

Garrigues-Baixauli, J. (2017b). Wave-Particle Duality as a Classic Phenomenon. Int. Sci. Res. Org. J. (ISROJ), 2(1).

Garrigues-Baixauli, J. (2018). The Origin of Long-Range Forces. International Journal of Science and Research (IJSR), 7(2).

Griffiths, D. J. (2015). Introduction to Quantum Mechanics (2nd ed.). Pearson Prentice Hall.

Haug, E. G. (2014). Unified Revolution: New Fundamental Physics. Oslo, E.G.H. Publishing.

Haug, E. G. (2016). The gravitational constant and the Planck units. A simplification of the quantum realm. Physics Essays, 29(4).

Haug, E. G. (2017). Can the Planck Length Be Found Independent of Big G? Applied Physics Research, 9(6), 58-66.

Haug, E. G. (2017a). Can the Planck length be found independent of big G? Applied Physics Research, 9(6).

Haug, E. G. (2017b). The ultimate limits of the relativistic rocket equation. The Planck photon rocket. Acta Astronautica, 136.

Haug, E. G. (2018). Revisiting the Derivation of Heisenberg's Uncertainty Principle: The Collapse of Uncertainty at the Planck Scale. Preprints, 2018050258.

Hawking, S. (1971). Gravitationally collapsed objects of very low mass. Monthly Notices of the Royal Astronomical Society, 152.

He et al. (2017). Chiral Majorana fermion modes in a quantum anomalous Hall insulator-superconductor structure. Science, 357, 294-299.

Heisenberg, W. (1943). Die Leobachtbaren Grossen in der Theorie der Elemntarteilchen. Z. Phys., 120, 513-538.

Heisenberg, W. (1957). Quantum Theory of Fields and Elementary Particles. Rev. Mod. Phys., 29, 269-278.

Huang, K. (1952). On the Zitterbewegung of the Dirac Electron. Am. J. Phys., 20, 479.

Hudson et al. (2011). Improved measurement of the shape of the electron. Nature, 473, 493-496.

Jennison, R. C. (1979). What is an Electron? Wireless World, 28(1522), 42-47.

Khrennikov, A. (2004). EPR-Bohm experiment, interference of probabilities, and imprecision of time.

Lorentz, H. A. (1904). Proc. Acad. Sci. Amsterdam, 6, 809.

Macgibbon, J. H. (1987). Can Planck-mass relics of evaporating black holes close the universe? Nature, 329.

Meessen, A. (1999). Spacetime Quantization, Elementary Particles and Cosmology. Foundations of Physics, 29 , 281-316.

Meessen, A. (2011). Space-Time Quantization, Elementary Particles and Dark Matter.

Mohr, P. J., Taylor, B. N., \& Newell, D. B. (2016). Rev. The 2014 CODATA Recommended Values of the Fundamental Physical Constants.

Motz, L. (1962). Gauge invariance and the structure of charged particles. Il Nuovo Cimento, 26(4).

Motz, L. (1971). The quantization of mass. Rutherford Observatory, Columbia University.

Motz, L., \& Epstein, J. (1979). The gravitational charge 1/2 (hc)1/2 as a unifying principle in physics. Il Nuovo Cimento, 51(1).

Neumaier, A. (2018). A theoretical physics FAQ: B2 Are electrons pointlike/structureless? Retrieved from https://www.mat.univie.ac.at/ neum/physfaq/physics-faq.html 
Obermair, G. M. (2013). Primordial Planck mass black holes (ppmbhs) as candidates for dark matter? Journal of Physics, Conference Series, 442.

Odom, B., Hanneke, D., D’Urso, B., \& Gabrielse, G. (2006). New Measurement of the Electron Magnetic Moment Using a One-Electron Quantum Cyclotron. Phys. Rev. Lett.

Padmanabhan, T. (1985a). Physical Significance of Planck Length. Ann. Phys., 165(1), 38-58.

Padmanabhan, T. (1985b). Planck Length As The Lower Bound To All Physical Length Scales. Gen. Rel. Grav., 17(3), 215-221.

Parson, A. L. (1915). A Magneton Theory of the Structure of the Atom. Smithsonian Miscellaneous Collections, $65,2-80$.

Penrose, R. (2004). The road to reality. Alfred A. Knopf.

Planck, M. (1899). Naturlische Masseinheiten. Der Königlich Preussischen Akademie Der Wissenschaften, 479.

Planck, M. (1906). Vorlesungen über die Theorie der Wärmestrahlung (p. 163). Leipzig: J.A. Barth.

Poincaré, H. (1905). Acad. Sci. Paris, 140, 1504.

Poincaré, H. (1906). Rendiconti Circ. Matemat. Palermo, 21, 129.

Prasannakumar, S., Krishnaveni, S., \& Umesh, T. K. (2012). Determination of rest-mass energy of the electron by a Compton scattering experiment. European Journal of Physics, 33(1).

Prokhovnik, S. J. (1967). The Logic of Special Relativity. (Cambridge University Press), Cambridge.

Rivas, M. (2001). Kinematical Theory of Spinning Particles. Kluwer, Dordrecht.

Sebens, C. T. (2018). How Electrons Spin. University of California, San Diego.

Smolin, L. (2004). Atoms of Space and Time. Sci. Am., 290(1), 66-75.

Sprenger, M., Nicolini, P., \& Bleicher, M. (2012). Physics on the Smallest Scales - An Introduction to Minimal Length Phenomenology. Eur. J. Phys., 33(4), 853-862.

Susskind, L. (2008). The Black Hole War. Hachette Book Group US.

Tomonaga, S.-I. (1997). The Story of Spin (p. 35). University of Chicago Press.

Uhlenbeck, G. E., \& Goudsmit, S. (1925). Die Naturwissenschaften, 13, 953-954.

Weinberg, S. (1993). Dreams of a Final Theory, the search for the fundamental laws of Nature, 148.

Wilczek, F. (2008). The Lightness Of Being. Basic Books.

William et al. (2017). Precision Measurement of the Electron's Electric Dipole Moment Using Trapped Molecular Ions. Phys. Rev. Lett., 119, 153001.

Williamson, J. G., \& van der Mark, M. B. (1997). Is the electron a photon with toroidal topology? Annales de la Fondation Louis de Broglie, 22(2), 133.

\section{Copyrights}

Copyright for this article is retained by the author(s), with first publication rights granted to the journal.

This is an open-access article distributed under the terms and conditions of the Creative Commons Attribution license (http://creativecommons.org/licenses/by/4.0/). 